\title{
Intentional Rechallenge and the Clinical Management of Drug-Related Problems
}

\author{
Ronald Meyboom
}

Published online: 9 March 2013

(C) Springer International Publishing Switzerland 2013

\section{Background}

The use of medicines can lead to a wide variety of different problems. Taken together, drug-related problems are commonly encountered in medical practice and often therapeutic interventions of some sort are needed. Many such interventions may have a pragmatic or experimental side; established protocols are often not available.

Prominent drug-related problems are adverse reactions, drug interactions and therapeutic failure [1]. In addition to drug discontinuation, a characteristic intervention associated with—often suspected-adverse drug reactions, is a re-exposure test: an intentional rechallenge. Such a re-exposure can, even in small amounts, be dangerous, however, and acute and fulminant reactions may occur. A rechallenge test may raise major clinical, scientific and ethical concerns. In life-threatening adverse reactions, such as toxic epidermal necrolysis, Stevens-Johnson Syndrome or agranulocytosis (Schultz disease), a rechallenge is almost always contraindicated. Sometimes a skin test or laboratory finding can help, such as a patch test, RAST (radioallergosorbent) test, a lymphocyte stimulation test or other evidence of drug-dependent immunity.

On the other hand, refraining from a-careful and monitored-rechallenge test and the persistence of uncertainty may, in a different way, put a patient at serious risk.

Some signs during the original adverse event may be predictive of a future serious response, e.g. urticaria, wheezing, angioedema (risk signs for a future anaphylactic

R. Meyboom $(\square)$

Division of Pharmacoepidemiology

and Clinical Pharmacology, University of Utrecht,

P.O. Box 80082, 3508 TB Utrecht, The Netherlands

e-mail: r.h.b.meijboom@uu.nl reaction), eosinophilia, fever, abnormal liver tests (increased aminotransferase levels) or low blood counts. On the other hand, many other adverse drug effects are less serious and careful reintroduction of the drug, usually at a lower dose, may be safe, beneficial and satisfactory for the patient.

Drug rechallenge and 'dechallenge', in the true sense of the words, are planned, prospective experiments, comparable with an 'N-of-1 clinical trial' [2]. The prime reason for a rechallenge is the acquisition of additional information as needed for the (future) treatment and well-being of the patient. Abandoning the future use of a drug or group of drugs, or therapeutic dilemmas when a treatment is urgently needed may be disastrous to the patient. For such reasons, further evidence regarding the likelihood of involvement of a suspected drug may be of substantial importance. In addition, rechallenge observations-when made available-may contribute to science and create further knowledge, and be of value to other (future) users of the drug, as well as to companies and regulators (and lawyers and judges).

For many drugs and adverse reactions there is little or no information about the best way forward in the case of a rechallenge. For only a few drugs, for instance $\beta$-lactam antibiotics, there is a substantial body of published knowledge on how to proceed in suspected hypersensitivity. In a hospital setting, expertise may have accumulated in specialized units, for instance dermatology, clinical pharmacology or allergology.

\section{Flowchart}

With special emphasis on intentional re-exposure, in this issue of Drug Safety Stanulovic et al. [3] provide guidance on the often complex process of decision making in the 
clinical management of patients with (suspected) adverse drug reactions, using a flowchart with questions and considerations to serve as a structure for reasoning. Crucial questions are as follows. How dangerous is the re-exposure and how can risk be mitigated? How important is this drug for this patient, now or in the future? How likely is it that this patient is intolerant to this drug? Which (other) drugs are likely to be contraindicated in the future? As always in pharmacotherapy, the ultimate question here is the weighing of benefit and risk: do patient and doctor think that the importance of the expected information outweighs the risk of the rechallenge. This flowchart has four steps, all capturing a number of criteria and considerations which together guide the clinician in a fairly comprehensive way through a structured process of balanced decision making.

\section{Situations that May Influence a Rechallenge}

There are, however, many different types of drugs, adverse reactions and patients. Different situations may strongly influence the way a dechallenge or rechallenge should be done and how the outcome should be interpreted. In particular, when done inappropriately, a rechallenge experiment may not necessarily lead to an objective, exact and unambiguous outcome. Examples of different types of drugs and adverse reactions that may require different rechallenge strategies are shown in Table 1. Likewise adapted flow charts may be needed. In addition to the active ingredients in a medicinal product, in hypersensitivity reactions the possible involvement of a colouring agent or other excipients may need to be considered.

Dechallenge and rechallenge are often complex clinical experiments and both need a plan in order to obtain the best possible evidence. An acute hypersensitivity reaction is often an emergency, and all too often all drugs are stopped immediately and simultaneously, leaving both the doctor and the patient in uncertainty with regard to the factual cause of the adverse reaction and also to the question of which drug or drugs will be contraindicated in the future. The next step, resuming the treatment of the patient with the same or another drug, and also taking into account which parts of the patient's treatments are clinically most needed and which drugs are most likely to be safe (although this is often uncertain), is closely related to the rechallenge question. Firstly, the most needed and least suspect drugs will, one after another, be (re)started and, in the absence of a relapse, the most likely causative drug may indirectly be identified, even though it is not itself 'rechallenged'. This procedure, which may also produce, indirectly, evidence with regard to the most likely cause of the adverse reaction, might be regarded as 'a rechallenge by exclusion'.
Table 1 Examples of different types of medicines and adverse reactions that may, in rechallenge experiments, need different strategies

Medicines

Small molecule drugs

Biopharmaceuticals and blood products

Antiretroviral drugs

Oncolytics and immunosuppressants

Contrast dyes, diagnostics, radiopharmaceuticals

Vaccines (revaccination); desensitization vaccines

Adverse reactions [1]

'Type A': These are predominantly pharmacological effects. For such reactions, a rechallenge test is designed based on pharmacological criteria and involves (low) dose within therapeutic range (or high dose in case of a therapeutic failure). Drug interactions are a subtype of Type A adverse reactions. A careful rechallenge is not necessarily dangerous

'Type B': These are hypersensitivity reactions. For such reactions, a rechallenge test is designed based on immunological (or metabolic) criteria and with a (very) low dose only. Often the underlying mechanism is uncertain ('idiosyncratic').

Rechallenge is potentially dangerous. Cross hypersensitivity between drugs may occur

'Type C': These reactions often have no suggestive time course and may follow neither pharmacological nor immunological principles. For example, increased risk of acquiring a 'natural' disease (e.g. myocardial infarction, pulmonary embolism), or increased frequency of a recurrent but transient event (e.g. more frequent migraine or convulsions).

A rechallenge may be inconclusive or not practicable

In predominantly pharmacological ('Type A') adverse effects the rechallenge dose often needs to be in the therapeutic range; if the dose and duration of use are inappropriate the rechallenge outcome may be worthless. In the case of a hypersensitivity reaction, the response follows the rules of the immune system or of the underlying error of metabolism (e.g. haemolysis in glucose-6-phosphate dehydrogenase deficiency); a (semi)therapeutic dose may be disastrous. In many serious but rare adverse reactions, however, the mechanism is uncertain or unknown.

Adverse events may, in different patients, have a different pattern. Patients with infectious mononucleosis (or lymphatic leukaemia), for example, usually get a rash after taking ampicillin or amoxicillin but do not so after recovery of the primary disease. Allergic reactions to antibiotics are common during a chemotherapy induction course in leukaemia patients but hypersensitivity may not necessarily continue when the patient is in remission. Thrombocytopenia caused by a first measles vaccination does not, as a rule, recur after the second vaccination (a 'false negative rechallenge').

If a rechallenge test is thought to be clinically needed, it may, in addition to the informed consent of the patient, be recommendable (for the protection of the patient and the 
physician) to first consult the hospital's ethics committee (for instance for the prevention of possible malpractice or legal reasons).

In the field of pharmacovigilance, observations derived from dechallenge or rechallenge often play an important role, notably in causality assessment. A dechallenge may be difficult to interpret, for example if the follow-up period has been (too) short. An irreversible event should not have a 'positive dechallenge' but a 'negative' one (i.e. no recovery on stopping the drug). A case history with a 'positive rechallenge' is commonly thought of as a proofpositive case report. The scientific evidence contained in case reports may be modest or inconclusive, however, and the interpretation of the data is often difficult or uncertain. A positive rechallenge does not always provide proof; a negative rechallenge does not always refute the suspicion. An increase in aminotransferases upon rechallenge to a drug, for instance, may, in spite of a suggestive time course, reflect the natural course of an unrecognized underlying hepatic disorder. Also, a negative rechallenge may not be conclusive. It may occur when the dose has simply been too low, or exposure or follow-up has been too short. An anaphylactoid reaction, for example to morphine, may not recur on rechallenge. Even Stevens-Johnson Syndrome may not always relapse on re-exposure to the original drug. After some time a change in immune regulation may inhibit or remove a previous drug allergy.

Deliberate, planned, prospective rechallenge experiments are rare and, as Stanulovic et al. [3] emphasize, rarely justified; published experience is limited. What happens most in clinical practice is the inadvertent re-exposure of patients to a drug after a prior adverse event, i.e. an 'unintentional rechallenge', for instance because the drug was not yet suspected or had erroneously been restarted.

In a pharmacovigilance database, case reports with a recorded 'positive rechallenge' may often not really represent prospective planned re-exposures. Often a recorded 'rechallenge positive' may refer to the situation that the patient had previously experienced a similar (often weaker) response to the drug; this might be called a 'historical rechallenge'. In such cases, often the event described in the case report had itself been the 'rechallenge' episode, which had unmasked the causative drug. Obviously the persuasiveness of such reports may be less than in the case of a well-planned and documented rechallenge observation.

\section{Observations}

The rechallenge flowchart proposed by Stanulovic et al. [3] is welcome and needed, and seems well designed. Its value and practicability, however, has not yet been demonstrated. In this phase it is primarily a 'way of thinking', a structure assisting in rational reasoning and careful decision making. A trial of its usefulness will be the next step. Obviously the harmonization of medical interventions is often worthwhile. It is possible, however, that the flowchart as it is may be particularly useful for the group of 'small molecule' drugs but may be less appropriate for other types of medicines (see Table 1). More experience is needed to find out whether adapted versions of the scheme are needed for other types of adverse reactions.

As mentioned by Stanulovic et al. [3], sometimes prospective clinical re-exposures are not done in one patient but in a series of patients with a particular (suspected) adverse reaction. In such studies the ethical situation is similar to that in therapeutic clinical trials and apply the rules of Good Clinical Trial Practice. In addition to the judgement of the ethics committee and the patients' informed consent, in such trials this flowchart may also be of value in the assessment of the acceptability of the risk or harm to individual trial participants.

Acknowledgments I thank Dr. Eugène van Puijenbroek for his valuable comments. No sources of funding were used to prepare this commentary. The author has no conflicts of interest that are directly relevant to the content of this commentary.

\section{References}

1. Meyboom RHB, Lindquist M, Egberts ACG. An ABC of drugrelated problems. Drug Saf. 2000;22:415-23.

2. Gabler NB, Duan N, Vohra S, Kravitz RL. N-of-1 trials in the medical literature: a systematic review. Med Care. 2011;49(8): 761-8.

3. Stanulovic V, Venegoni M, Edwards B. Intentional rechallenge: does the benefit outweigh the risk? Drug Saf. 2013. doi:10.1007/ s40264-013-0020-3. 